
AS AN ALTERNATIVE TO PARENTERAL NUTRITION FOR OVARIAN CANCER PATIENTS WITH INOPERABLE MALIGNANT BOWEL OBSTRUCTION

${ }^{1}$ Agnieszka Michael, ${ }^{2}$ Lindsey Allan, ${ }^{3}$ Kate Bennett-Eastely, ${ }^{4}$ Rebecca Herbertson, ${ }^{5}$ Simon Skene. ' University of Surrey; School of Biosciences and Medicine; ${ }^{2}$ Royal Surrey Foundation Trust; Oncology; ${ }^{3}$ University of Surrey; Clinical Trials Unit; ${ }^{4}$ Brighton and Sussex Hospital; Oncology; ${ }^{5}$ University of Surrey; Clinical Trials Unit, University of Surrey; School of Biosciences and Medicine

\subsection{6/ijgc-2020-ESG0.144}

Introduction/Background Inoperable bowel obstruction (IBO) occurs in up to $50 \%$ of patients diagnosed with ovarian cancer. Nutrition support for patients with IBO is challenging. Parenteral feeding (PN) is the recommended route for patients with a prognosis of $>2$ months, however there is little evidence that it improves quality of life and the cost of it is very high. If $\mathrm{PN}$ is not available patients are frequently discharged home from hospital with sips of clear fluids only. Management of inoperable bowel obstruction remains a major challenge and clear guidelines are needed.

Elemental diet (ED) is a liquid diet that contains proteins in the form of amino acids, fats in the form of medium chain triglycerides, vitamins and trace minerals. ED is almost completely absorbed in the upper small intestine.

Methodology The primary objective of the study was to establish if ED can be used as an alternative to home PN in patients with IBO. The secondary aim was to examine the impact of ED on quality of life. The primary endpoints of the study were acceptability and tolerability of ED with respect to taste, and incidence of vomiting and pain. The secondary endpoints included the number of patients alive at the end of the study, quality of life, nutritional intake, and the number of women who can tolerate ED and subsequently be treated with palliative chemotherapy (as per standard of care).

Results 29 women with IBO caused by metastatic ovarian cancer were recruited into the EDMONd study. Of those 8 could not complete the trial due to disease progression, and 2 had missing data that was deemed irretrievable, leaving 19 patients who contributed data to the primary endpoint analysis. The mean age of the patients who continued the trial was 68 (SD 12.5). Preliminary analysis shows that $68.4 \%$ of patients met the primary endpoint and tolerated ED; the ED did not worsen the vomiting or pain as measured by Memorial Symptoms Assessment Scale. At baseline $72 \%$ of patients experienced vomiting and this number reduced to $28 \%$ by the end of week 1 of the study and to $23.5 \%$ by the end of week 2 . $96 \%$ of patients reported pain at baseline and this proportion reduced to $72 \%$ and $76 \%$ by the end of week 1 and 2 respectively.

Conclusion ED is well tolerated by patients with IBO and can provide an acceptable feeding option for this group of patients.

Disclosures The study was funded by Target Ovarian Cancer charity.

The author received educational grants from BMS, GSK, IPSEN, NOVARTIS, PFIZER

CLOVIS, TESARO, ESAI;

Advisory boards: CLOVIS, ESAI, IPSEN, ROCHE, TESARO

\section{ORAL METRONOMIC CYCLOPHOSPHAMIDE IN RECURRENT OVARIAN CANCER: A SINGLE CENTRE EXPERIENCE}

${ }^{1}$ Daniela Attianese, ${ }^{1}$ Enrico Badellino, ${ }^{1}$ Michela Villa, ${ }^{2}$ Marco Bellero, ${ }^{1}$ Roberta Massobrio, ${ }^{1}$ Nicoletta Biglia, ${ }^{1}$ Annamaria Ferrero. 'Mauriziano Hospital; Academic Department Gynaecology; ${ }^{2}$ Mauriziano Hospital; Hospital Pharmacy

\subsection{6/ijgc-2020-ESG0.145}

Introduction/Background Oral metronomic cyclophosphamide (OMC) consists in the chronic administration of low, usually daily, doses of chemotherapy. The effective reduction of tumour growth, oral administration, low toxicity profile and low cost benefit women with relapsed ovarian cancer, especially heavily pretreated patients. We retrospectively evaluated the outcome of patients treated with OMC for recurrent ovarian cancer.

Methodology We selected patients treated with OMC $(50 \mathrm{mg}$ daily) from 2016 to 2020 at the Academic Department Gynaecology, Mauriziano Hospital, Torino, Italy. Progression free survival (PSF) and toxicities profile were evaluated.

Results Thirty-five patients were analyzed. 28 (87\%) had FIGO stage III and IV disease at diagnosis and 59\% had received $\geq 4$ previous lines. Average age was 68 years (range 47-88). Before starting OMC 16\% had ECOG 0, 65\% ECOG 1 and 19\% ECOG 2. Median PFS was 5 months. PFS was $\geq 6$ months in $33 \%$ of patients, $\geq 12$ months in $13 \%$ and $\geq 18$ months in 7\%. 52\% experienced clinical benefit in terms of symptoms reduction. $3 \%$ of discontinuation for side effects and no G3-4 hematological toxicities reflected a low toxicity profile. Only nausea and fatigue G1-G2 were reported in $4(12 \%)$ and $9(28 \%)$ cases, respectively.

Conclusion OMC could be a feasible alternative therapy for recurrent ovarian cancer leading to an acceptable clinical response with a low toxicity profile, even if patients are heavily pretreated and with a suboptimal performance status.

Disclosures Authors have no conflict of interest.

\section{NIRAPARIB OUTCOMES IN BRCA WILD-TYPE PLATINUM SENSITIVE RECURRENT OVARIAN CANCER: A COMPARISON OF REAL-WORLD DATA TO THE NOVA TRIAL}

Douglas Cartwright, Patricia Roxburgh, Barbara Stanley, Jennifer Brown, Alistair Mclaren Sarah Coulter, Carla Forte, Ros Glasspool. Beatson West of Scotland Cancer Centre

\subsection{6/ijgc-2020-ESG0.146}

Introduction/Background The PARP inhibitor (PARPi), niraparib, is EMA approved for maintenance treatment in platinum-sensitive recurrent ovarian cancer. The eligibility criteria set out in niraparib licence are less stringent than those in the NOVA trial, therefore outcomes may be different in the realworld to those observed in trial patients. ${ }^{1}$ The optimal management of patients after progression on PARPi is unknown and the relationship between platinum free interval and probability of response to platinum may be modified after PARPi therapy. To investigate this, we performed a retrospective analysis of real-world niraparib use and compared it to the NOVA trial.

Methodology Data was collected retrospectively for all women receiving maintenance niraparib for BRCA wild-type, platinum 
sensitive relapsed ovarian cancer between June 2017 and September 2019. Response to prior platinum, median progressionfree survival (mPFS) after 1st and subsequent platinum, number of cycles of PARPi, dose, haematological toxicities, and PFS2I (start of subsequent therapy to physician assessed progression or death) were obtained through electronic records.

Results 37 patients received Niraparib in this timeframe. Median follow up was 16 months (range 5.7-37 months). Demographics were similar to previously published cohorts, however, only $11 \%(n=4)$ had a complete response (CR) to prior platinum therapy and 59\% $(n=22)$ had a partial response in comparison to $50 \% \mathrm{CR}$ and $50 \% \mathrm{PR}$ in the NOVA trial1. 35 (95\%) of patients had progressed on niraparib at the time of data collection. The mPFS on niraparib was 4.4 months (95\% CI 3.7 - 6.7 months) in comparison to 9.3 months in the NOVA study. Patients who met the NOVA trial radiological and serological response criteria, had a mPFS at 5.1 months $(n=19)$ compared to 3.9 months $(n=18)$. Dosing and toxicity data will be reported in full at the meeting. 31 patients received subsequent therapy, 19 (61\%) were treated with paclitaxel, $9(29 \%)$ were treated with platinum-based chemotherapy. Median PFS2I was 5.8 months for platinum sensitive disease and 3.5 months for platinum resistant disease.

Conclusion The real-world outcomes for niraparib treatment are worse than observed in the NOVA trial. Patients who meet NOVA trial eligibility criteria have better outcomes, however, these results are still inferior to those reported in the trial. Post PARP outcomes are poorer than expected in both platinum sensitive and platinum resistant settings. Strategies to effectively treat PARPi resistant disease are urgently needed.

Disclosures Dr R Glasspool: Grant funding for clinical trials from Boehringer Ingelheim, Lilly/Ignyta and Clovis. Consultancy fees, travel support and/or speaker fees for AstraZeneca, GSK/Tesaro, Clovis, Immunogen and Sotio. Site PI for studies sponsored by AstraZeneca, GSK/Tesaro, Clovis, Immunogen, Lilly and Pfizer.

\section{REFERENCE}

1. Mirza MR, et al. Niraparib Maintenance Therapy in Platinum-Sensitive, Recurrent Ovarian Cancer. N Engl J Med 2016;375:2154-64.

\section{PHASE 1B TRIAL OF MONALIZUMAB (NKG2A INHIBITOR) PLUS DURVALUMAB: SAFETY AND EFFICACY IN PATIENTS WITH METASTATIC OVARIAN, CERVICAL, AND MICROSATELLITE-STABLE ENDOMETRIAL CANCERS}

${ }^{1}$ Susana Banerjee, ${ }^{2}$ Ana Oaknin, ${ }^{3}$ Inmaculada Sanchez-Simon, ${ }^{4}$ Alfonso Cortes Salgado, ${ }^{5}$ Sandip Pravin Patel, ${ }^{6}$ Amit Oza, ${ }^{7}$ Mayukh Das, ${ }^{7}$ Panagiotis Kourtesis, ${ }^{7}$ Maria Libera Ascierto, ${ }^{8}$ Jennifer R Diamond. 'Royal Marsden NHS Foundation Trust and Institute of Cancer Research; ${ }^{2}$ Hospital Vall D'hebron, Vall D'hebron Institute of Oncology (Vhio); ${ }^{3}$ Hospital Universitario Virgen; ${ }^{4}$ Hospital Universitario Ramón; ${ }^{5}$ Ucsd Moore Cancer Center; ${ }^{6}$ Princess Margaret Cancer Centre; ${ }^{7}$ Astrazeneca; ${ }^{8}$ University of Colorado Cancer Center

\subsection{6/ijgc-2020-ESG0.147}

Introduction/Background The immune checkpoints NKG2A and programmed cell death-1 (PD-1) are expressed on both tumour-infiltrating natural killer (NK) and CD8 $+\mathrm{T}$ cells in several cancer types, and are implicated in reducing antitumor immune response. To evaluate whether dual targeting of nonredundant checkpoint pathways (NKG2A/HLA-E and PD-1/ PD-L1) may enhance antitumour immunity, the combination of monalizumab and durvalumab is being assessed in a Phase $1 \mathrm{~b}$ expansion study in multiple solid tumours
(NCT02671435). Here we report safety and efficacy results in patients with ovarian, cervical or microsatellite-stable (MSS) endometrial cancer.

Methodology Eligible patients had advanced recurrent or metastatic high-grade serous epithelial ovarian cancer or cervical cancer (patients in each cohort could have received up to 2 prior lines of systemic therapy) or MSS endometrial cancer (patients could have received up to 3 prior lines of systemic therapy), with Eastern Cooperative Oncology Group performance status $0-1$. Patients received monalizumab $750 \mathrm{mg}$ Q2W and durvalumab $1500 \mathrm{mg}$ Q4W for up to 3 years until unacceptable toxicity or confirmed progression. The primary endpoint was safety and tolerability; secondary endpoints included antitumour activity.

Results Between March 2017 and March 2019, 40 patients with ovarian cancer (age range, 42-75 years), 16 patients with cervical cancer (age range, 32-79 years) and 40 patients with MSS endometrial cancer (age range, 45-79 years) were enrolled. Rates of treatment-related adverse events (AEs) were generally similar across cohorts (table 1 ). There were no grade 5 AEs and no events leading to discontinuation of monalizumab or durvalumab. Objective responses were seen only in ovarian cancer (table 2): among the 37 evaluable patients, 2 (5.4\%) had confirmed partial responses and 10 (27.0\%) had stable disease (SD) including $6(16.2 \%)$ with disease control at 24 weeks (DCR24). Median progression-free survival (mPFS) was 1.8 months and median overall survival (mOS) was 16.7 months. Six $(37.5 \%)$ of the 16 evaluable patients with cervical cancer had SD; mPFS was 2.0 months and mOS was 8.6 months. Fifteen (38.5\%) of the 39 evaluable patients with MSS endometrial cancer had SD, including 5 (12.8\%) with DCR24; mPFS was 1.8 months and mOS was 10.7 months. Conclusion Monalizumab plus durvalumab treatment had manageable safety in all cohorts. Modest clinical activity was demonstrated in recurrent ovarian cancer, whereas activity in cervical and MSS endometrial cancers was minimal. Further understanding of dual immune-checkpoint targeting is required.

Abstract 518 Table 1 Safety in patients with ovarian, cervical or MSS endometrial cancer

\begin{tabular}{|l|c|c|c|}
\hline Safety & $\begin{array}{c}\text { Ovarian } \\
\text { cancer } \\
(\mathbf{N = 4 0 )}\end{array}$ & $\begin{array}{c}\text { Cervical } \\
\text { cancer } \\
(\mathbf{N}=16)\end{array}$ & $\begin{array}{c}\text { MSS endometrial } \\
\text { cancer } \\
(\mathbf{N}=40)\end{array}$ \\
\hline At least one monalizumab-related AE, $n(\%)$ & $23(57.5)$ & $9(56.3)$ & $24(60.0)$ \\
\hline At least one durvalumab-related AE, $n(\%)$ & $24(60.0)$ & $9(56.3)$ & $26(65.0)$ \\
\hline At least one monalizumab-related SAE, $n(\%)$ & $3(7.5)$ & $2(12.5)$ & $3(7.5)$ \\
\hline At least one durvalumab-related SAE, $n(\%)$ & $4(10.0)$ & $2(12.5)$ & $2(5.0)$ \\
\hline Death (grade 5 severity), $n(\%)$ & 0 & 0 & 0 \\
\hline $\begin{array}{l}\text { At least one event leading to monalizumab } \\
\text { discontinuation, } n(\%)\end{array}$ & 0 & 0 & 0 \\
\hline $\begin{array}{l}\text { At least one event leading to durvalumab } \\
\text { discontinuation, } n(\%)\end{array}$ & 0 & 0 & 0 \\
\hline
\end{tabular}

Abstract 518 Table 2 Efficacy in patients with ovarian, cervical or MSS endometrial cancer

\begin{tabular}{|l|c|c|c|}
\hline Efficacy & $\begin{array}{c}\text { Ovarian } \\
\text { cancer } \\
(\mathrm{N}=37)\end{array}$ & $\begin{array}{c}\text { Cervical } \\
\text { cancer } \\
(\mathrm{N}=16)\end{array}$ & $\begin{array}{c}\text { MSS endometrial } \\
\text { cancer } \\
(\mathrm{N}=39)\end{array}$ \\
\hline Complete response, $\mathrm{n}(\%)$ & 0 & 0 & 0 \\
\hline Partial response, $\mathrm{n}(\%)$ & $2(5.4)$ & 0 & 0 \\
\hline Stable disease, $\mathrm{n}(\%)$ & $10(27.0)$ & $6(37.5)$ & $15(38.5)$ \\
\hline Objective response rate, $\mathrm{n}(\%)$ & $2(5.4)$ & 0 & 0 \\
\hline Disease control rate at 24 weeks, $\mathrm{n}(\%)$ & $6(16.2)$ & 0 & $5(12.8)$ \\
\hline Median progression-free survival (months) & 1.8 & 2.0 & 1.8 \\
\hline Median overall survival (months) & 16.7 & 8.6 & 10.7 \\
\hline
\end{tabular}

